\title{
Queratoquistes odontogénicos maxilares en paciente con síndrome de Gorlin. Descripción de un caso y revisión de la literatura
}

\section{Maxillary odontogenic keratocysts in a Gorlin síndrome patient: Case report and literatura review}

\author{
Andrés González F. ${ }^{1}$ Ignacio Sanhueza T. ${ }^{1}$, Pamela Elyette Benitez A. ${ }^{1}$, \\ Sheila Huerga M. ${ }^{2}$, Alba Larrea R. $^{3}$
}

${ }^{1}$ Servicio de Otorrinolaringología, Complejo Hospitalario de Navarra. Pamplona, España.

2Orden Hospitalaria de San Juan de Dios. Pamplona, España.

${ }^{3}$ Servicio de Anatomía Patológica, Complejo Hospitalario de Navarra. Pamplona, España.

Los autores declaran no tener conflictos de interés.

Recibido el 21 de noviembre de 2020. Aceptado el 31 de marzo de 2021.

Correspondencia: Andrés González F.

Calle Carmen $11,1^{\circ} \mathrm{A}$ Pamplona, (31001).

Navarra, España. Email: andresgonfer@hotmail.com

\section{Resumen}

Los queratoquistes maxilares son frecuentes en pacientes con síndrome de Gorlin. Su tratamiento es debatido por su alta tendencia a la recidiva. En los últimos años la cirugía endoscópica nasosinusal ha adquirido importancia en el manejo de esta patología. Exponemos en caso de un varón de 16 años afecto de este síndrome con queratoquistes maxilares donde se realiza un abordaje combinado, endonasal y transoral.

Palabras clave: Síndrome de Gorlin, queratoquiste, genética, patología nasosinusal

\begin{abstract}
Maxillary keratocysts are frequent in Gorlin Syndrome patients. Its treatment is discussed due to the high tendency to recurrence. In the last years the sinonasal endoscopic surgery has become an important tool in the management of this pathology. We report a 16 years old boy with Gorlin Syndrome and maxillary keratocysts treated with a trans-nasal endoscopic and intra-oral combined approach.
\end{abstract}

Keywords: Gorlin syndrome, keratocyst, genetics, sinonasal pathology.

\section{Introducción}

Los queratoquistes son lesiones agresivas localmente con alta prevalencia en el síndrome de Gorlin ${ }^{1-4}$. Son originadas en la lámina dental y se caracterizan por su alta agresividad, su rápido crecimiento y su capacidad de invadir estructuras próximas, incluyendo tejido óseo ${ }^{4}$. Tienen una alta tendencia a la recidiva, de modo que su tratamiento es un tema de continuo debate ${ }^{4}$. Describimos el caso de un paciente de 16 años con un síndrome de Gorlin con queratoquistes que ocupan ambos senos maxilares con una pieza molar impactada en el derecho, tratado con un abordaje combinado, endoscópico y transoral.

\section{Caso Clínico}

Varón de 16 años con síndrome de Gorlin confirmado genéticamente, con lesiones quísticas en ambos senos maxilares e impactación molar en el derecho. Ya habiendo sido intervenido por cirugía maxilofacial por otros queratoquistes mandibular y maxilares en dos ocasiones (Figuras $1 \mathrm{~A}$ y $1 \mathrm{~B}$, corresponden a estos quistes, antes de las primeras intervenciones), mediante drenaje de los mismos y posterior legrado de la mucosa (abordaje transalveolar para el quiste mandibular, y abordaje de Caldwell-Luc bilateral para los maxilares) sin otro tratamiento adyuvante; se solicita valoración para plantear tratamiento combinado del nuevo quiste. 


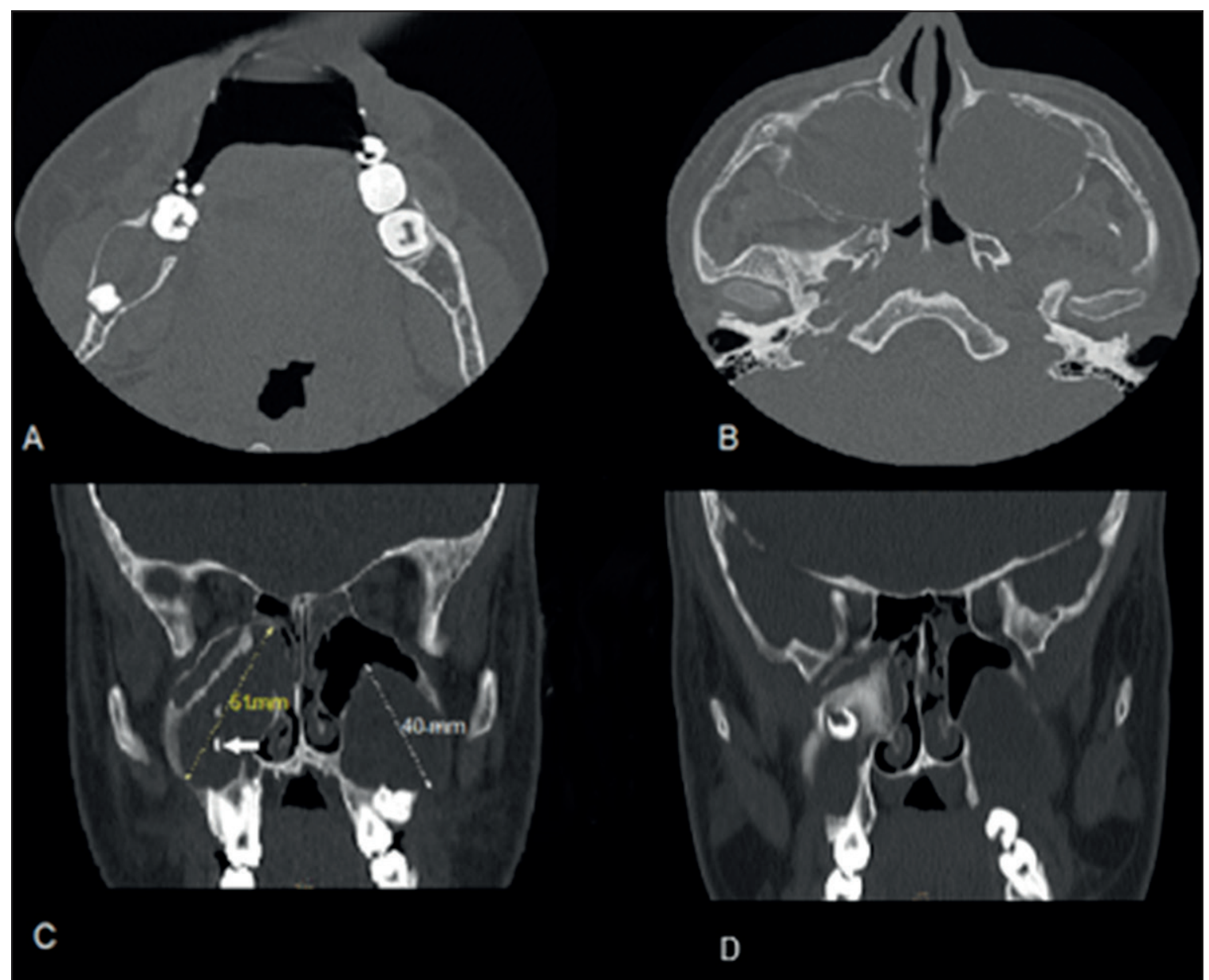

Figura 1. A y B: Tomografía computada (TC) de los queratoquistes mandibular y maxilares intervenidos por cirugía maxilofacial mediante drenaje y legrado de los mismos sin tratamiento adyuvante. C: Imagen coronal de TC previo al abordaje combinado con queratoquistes ocupando ambos senos maxilares. Se pueden apreciar lesiones hipodensas con un anillo radiopaco a su alrededor. El quiste derecho alcanza el techo etmoidal, presenta un diámetro máximo de $51 \mathrm{~mm}$ y en él se aprecia una pieza molar impactada señalada con la flecha. El quiste izquierdo tiene un diámetro máximo de $41 \mathrm{~mm}$. D: Imagen coronal del TC previo al abordaje combinado, posterior a la imagen en $C$, donde se identifica con claridad una pieza molar impactada dentro del quiste.

En el estudio radiológico prequirúrgico con tomografía computada de cavidades paranasales, se puede identificar una ocupación de ambos senos maxilares por queratoquistes con osteitis y disrupciones óseas amplias en sus paredes posterolaterales y anteriores. El quiste derecho alcanza el techo sinusal con $51 \mathrm{~mm}$ de diámetro y el izquierdo con $40 \mathrm{~mm}$ (Figura 1C), con, además, una pieza molar impactada en el derecho (Figura 1D).

Se decide un abordaje combinado, primero endoscópico y posterior Caldwell-Luc bilateral. Se decide ocluir el defecto óseo re- sultante con grasa abdominal. En quirófano se realiza en primera instancia una septoplastia endoscópica para acceder adecuadamente a ambos meatos medios. Posteriormente se realiza una antrostomía maxilar bilateral con límite posterior en rama ascendente del hueso palatino, superior en pared inferior de la bulla etmoidal, anterior en rama ascendente del maxilar e inferior en cornete inferior. A continuación, se drena el contenido del quiste, obteniendo un material amarillento. En el lado derecho, se identifican trabéculas óseas intrasinusales, correspondientes a las paredes 


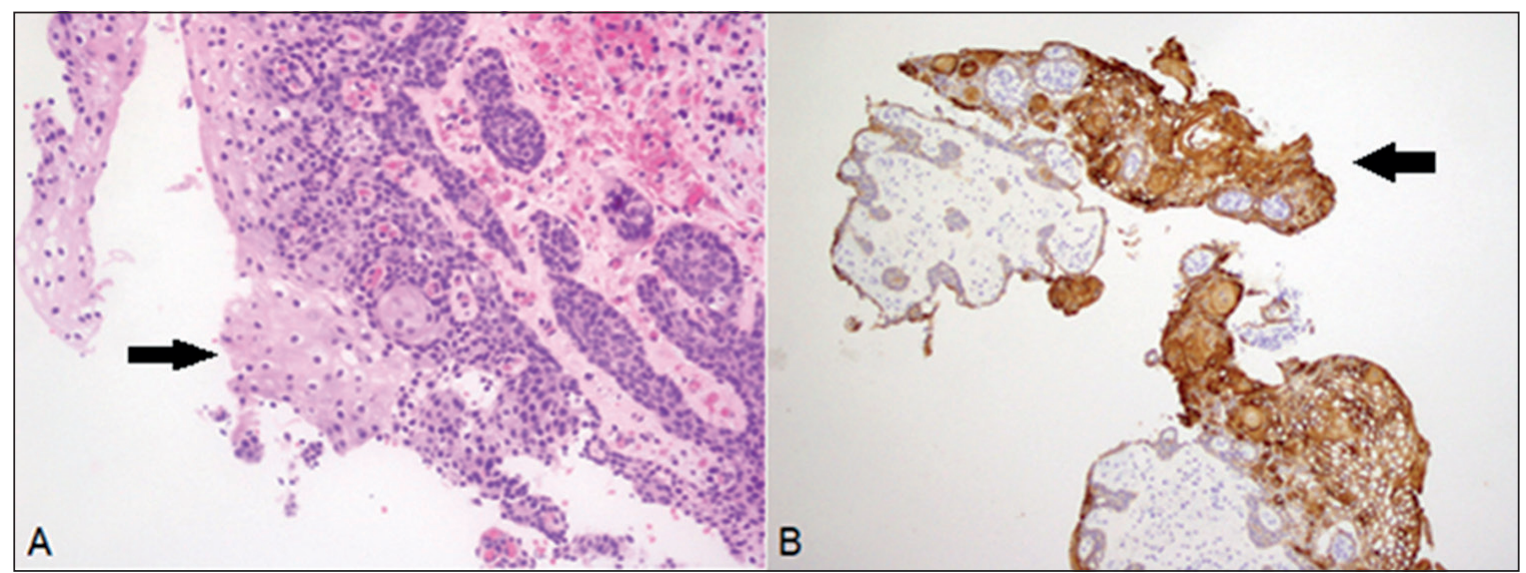

Figura 2. A y B: Cortes histológicos del quiste teñidos con hematoxilina y eosina y combinado de citoqueratina (citoqueratina AE1 y citoqueratina AE3) con queratina en la superficie. En $\mathbf{A}$, mediante la flecha señalamos el acúmulo de queratina, que simula el estrato córneo de la epidermis, con células anucleadas y filamentos de queratina (señalado con flecha). En B, el combinado de citoqueratina permite identificar el acúmulo de queratina en la mucosa del quiste (señalado con flecha).

del quiste, que son eliminadas, y se extrae el molar impactado. Posteriormente, se realiza un legrado prácticamente completo de la mucosa de ambas cavidades. Finalmente, se realiza un Caldwell-Luc bilateral para extracción de los restos epiteliales, creando un defecto de menor tamaño que en la intervención previa. Finalmente se sella el defecto óseo creado con el abordaje de Caldwell-Luc mediante la grasa abdominal previamente extraída, con el fin de separar las cavidades sinusales de la oral, y se realiza hemostasia.

El estudio posquirúrgico anatomopatológico confirma el queratoquiste bilateral (Figura 2). En el seguimiento se realiza una exploración semestral dirigida a ambos senos maxilares. Transcurridos los primeros 19 meses desde la intervención, no ha habido recidiva del queratoquiste.

\section{Discusión}

El síndrome de Gorlin es una enfermedad de herencia autosómica dominante relacionada con el gen patched ${ }^{1}$, localizado en el cromosoma 9q22-311 $3-5$, caracterizado por carcinomas basocelulares múltiples, queratoquistes maxilares y anomalías esqueléticas. Los queratoquistes maxilares suponen un criterio diagnóstico mayor en esta patología, ya que aparecen hasta en el 74\%-90\% de los pacientes afectos de este síndrome ${ }^{1}$.

Se trata de lesiones quísticas derivadas de la lámina dental ${ }^{4,6}$, agresivas localmente, con rápido crecimiento y tendencia a invadir tejidos próximos ${ }^{2,4}$. Son más frecuentes en mandíbula que en maxilar superior ${ }^{6,7}$, y en la mayor parte de los casos asintomáticos. No obstante, pueden manifestarse con dolor o hinchazón facial, en caso de sobreinfección. También se ha descrito la parestesia labial en la literatura ${ }^{7}$. El paciente descrito en el caso tenía episodios de dolor y cacosmia recidivantes.

Mediante estudios de imagen se pueden identificar como lesiones radiolucentes uniloculares o multiloculares, con un típico anillo radiopaco alrededor, y bordes festoneados ${ }^{2,5-7}$. Radiológicamente se distinguen cuatro variantes: queratoquistes de reemplazamiento, en la localización de un diente; de recubrimiento, abrazando un diente no erupcionado; externos, que no tienen relación con las estructuras dentarias; y colaterales, formados próximos a las raíces dentales ${ }^{7}$. El caso descrito corresponde a uno de los de recubrimiento, al abrazar el molar no erupcionado. Pueden asociarse con una impactación dentaria ${ }^{2,6}$, como ocurre en el caso presentado.

El tratamiento es tema de debate, por su alta tendencia a la recidiva ${ }^{4,6-9}$, siendo criterios que la favorecen la asociación con el síndrome 
de Gorlin, la resección incompleta del quiste, el crecimiento de un nuevo quiste a partir de restos epiteliales y el contacto de la cavidad con epitelio oral ${ }^{6,7}$. Entre las alternativas terapéuticas se encuentran la marsupialización y la enucleación como alternativas conservadoras; y ostectomía, crioterapia y aplicación de solución de Carnoy ( $1 \mathrm{~g}$ de cloruro férrico disuelto en $6 \mathrm{ml}$ de etanol al 95\%, $3 \mathrm{ml}$ de cloroformo y $1 \mathrm{ml} \mathrm{de}$ ácido acético glaciar) como técnicas más agresivas, y empleadas como técnicas adyuvantes, ya que parecen reducir el riesgo de recidiva ${ }^{4,6,8}$.

En relación a qué técnica conservadora emplear, no existe superioridad por ninguna de las alternativas; ni tampoco ninguna técnica adyuvante ha demostrado superioridad sobre las otras ${ }^{8}$. Desde hace alrededor de una década, la cirugía endoscópica nasosinusal se ha convertido en una herramienta interesante en el manejo de los quistes que ocupan el seno maxilar. Otorga beneficios como la reducción de la morbilidad que supone el abordaje oral, incluyendo alteraciones en la dentición, desarrollo de fístula oroantral, o patología nasosinusal crónica ${ }^{10}$. Además, reduce el riesgo de recidiva, al eliminar el contacto del quiste con la cavidad oral. No obstante, ante quistes localmente agresivos, la tasa de recidiva es similar a la obtenida con técnicas transorales ${ }^{10}$. Otra ventaja que aporta el abordaje endoscópico es que permite un seguimiento más sencillo del paciente en consulta, que es de gran interés dada la alta tasa de recidiva de la patología ${ }^{10}$.

Existen numerosas técnicas descritas, que tienen en común una antrostomía amplia que permita el acceso y la resección completa del quiste $^{10,11}$, pudiendo aplicarse posteriormente la solución de Carnoy, cauterizante, para eliminar los restos epiteliales y reducir el riesgo de recidiva ${ }^{4,11,12}$. Esta solución produce una rápida fijación local que puede aplicarse dentro del quiste, o sobre el lecho óseo ${ }^{12}$.

El abordaje combinado transoral y endoscópico ofrece buenos resultados en lo relacionado con morbilidad y reducción del riesgo de recidiva del queratoquiste maxilar; ya que permite realizar una descompresión del seno con el acceso nasosinusal y una eliminación de restos quísticos y de los dientes involucrados mediante un abordaje de Caldwell-Luc que no requiere de la creación de un gran defecto óseo en la pared sinusal ${ }^{13}$.
En el paciente descrito, se ha optado inicialmente por un abordaje endoscópico que permite reducir la morbilidad derivada del abordaje exclusivamente transoral ${ }^{10}$, y posteriormente un Caldwell-Luc para eliminar los restos epiteliales restantes, ya que la presencia de trabéculas intrasinusales dificulta la exéresis completa de los mismos por vía endoscópica. De este modo se elimina uno de los factores favorecedores de la recidiva ${ }^{6,7}$. Igualmente, el empleo de grasa abdominal aislando la cavidad oral de los senos maxilares reduce el riesgo de recidiva al eliminar otro de dichos factores favorecedores. Existen diversas alternativas para el cierre de esta comunicación, la elección de unas u otras depende de varios factores entre los que destacan el tamaño del, el lugar de asiento y la predilección del cirujano ${ }^{14}$. Las posibilidades son amplias, tanto colgajos locales, injertos como materiales inertes ${ }^{14}$. En el caso se prefiere el empleo de grasa abdominal, ya que, aunque los colgajos locales, como el colgajo de grasa yugal, son muy eficaces y ampliamente utilizados $^{14,15}$, se prefirió emplear tejido de otra localización, ya que había sido intervenido de varios carcinomas basocelulares en la región y recibe terapia fotodinámica. No hay reportes de empleo de grasa abdominal para el cierre de la comunicación oroantral, sin embargo, se trata de una localización accesible y disponible para tal fin; a pesar del inconveniente de la realización de una incisión extra en el procedimiento.

El colgajo pediculado de grasa yugal, aunque no se emplea en el caso, es una alternativa muy empleada y exitosa para el cierre de la comunicación oroantral. Su aporte vascular procede de varias ramas de la arteria maxilar. Debido a su rica irrigación, tiene un índice de fracaso muy bajo ${ }^{15}$. Existe bibliografía que respalda el abordaje endoscópico exclusivo, logrando tasas de éxito comparables al abordaje intraoral, con menor morbilidad ${ }^{10,11}$. Se recomienda un seguimiento semestral para valorar recidivas ${ }^{4}$.

\section{Conclusión}

Los queratoquistes maxilares son lesiones localmente agresivas con alta prevalencia en el síndrome de Gorlin. Su tasa de recurrencias es 
elevada; y por ello su tratamiento es debatido, ya que, a pesar de existir diferentes alternativas quirúrgicas, ninguna ha demostrado un índice de recidivas menor que las otras. El abordaje endoscópico ofrece unos resultados similares a los obtenidos por vía transoral en lo relacionado con recurrencias, con menor morbilidad. Puede acompañarse de tratamientos complementarios, como la aplicación de la solución de Carnoy, para la eliminación completa de restos epiteliales, o como un Caldwell-Luc que asegure la eliminación de dichos restos. Además, el acceso endoscópico facilita un seguimiento más sencillo del paciente.

\section{Bibliografía}

1. Palacios-Álvarez I, González-Sarmiento R, Fernández-López E. Gorlin Syndrome. Actas Dermosifiliogr. 2018;109(3):207-217. doi: 10.1016/j. ad.2017.07.018.

2. Lazaridou MN, Dimitrakopoulos I, Tilaveridis I, Iliopoulos C, Heva A. Basal cell carcinoma arising in association with a maxillary keratocyst in a patient with Gorlin-Goltz syndrome. Report of a case. Oral Maxillofac Surg. 2012;16(1):127-31. doi: 10.1007/ s10006-011-0270-0.

3. Agurto J, Mardones M, Núñez C. Síndrome de Gorlin-Goltz: A propósito de un caso clínico. Rev Otorrinolaringol Cir Cabeza Cuello. 2004: 64:230-236.

4. Kamil AH, Tarakji B. Odontogenic Keratocyst in Children: A Review. Open Dent J. 2016;10:117-23. doi: 10.2174/1874210601610010117.

5. Salmon B, Princ G, Wierzba CB. Kératokystes odontogènes dans le cadre d'un syndrome de Gorlin: à propos d'un cas clinique [Odontogenic keratocyst related to a Gorlin syndrome: a case report]. Arch Pediatr. 2008;15(4):406-9. doi: 10.1016/j. arcped.2008.01.009.

6. Singh M, Gupta KC. Surgical treatment of odontogenic keratocyst by enucleation. Contemp Clin Dent. 2010;1(4):263-7. doi: 10.4103/0976-
237X.76398.

7. Rajendra Santosh AB. Odontogenic Cysts. Dent Clin North Am. 2020;64(1):105-119. doi: 10.1016/j. cden.2019.08.002.

8. Ribeiro-Júnior O, Borba AM, Alves CAF, Gouveia MM, Deboni MCZ, Naclério-Homem MDG. Reclassification and treatment of odontogenic keratocysts: A cohort study. Braz Oral Res. 2017;31:e98. doi: 10.1590/1807-3107bor-2017. vol31.0098.

9. Antonoglou GN, Sándor GK, Koidou VP, Papageorgiou SN. Non-syndromic and syndromic keratocystic odontogenic tumors: systematic review and meta-analysis of recurrences. J Craniomaxillofac Surg. 2014;42(7):e364-71. doi: 10.1016/j. jcms.2014.03.020.

10. Marino MJ, Luong A, Yao WC, Citardi MJ. Management of odontogenic cysts by endonasal endoscopic techniques: A systematic review and case series. Am J Rhinol Allergy. 2018;32(1):40-45. doi: 10.2500/ajra.2018.32.4492.

11. Barry JY, Le CH, Baumann J, Skinker L, Chiu AG, Chang EH. Endoscopic resection of maxillary sinus keratocystic odontogenic tumors. Laryngoscope. 2016;126(10):2216-9. doi: 10.1002/lary.25920.

12. Díaz-Belenguer Á, Sánchez-Torres A, Gay-Escoda C. Role of Carnoy's solution in the treatment of keratocystic odontogenic tumor: A systematic review. Med Oral Patol Oral Cir Bucal. 2016;21(6):e689-e695. doi: 10.4317/medoral.21250.

13. Procacci P, Lanaro L, Molteni G, Marchioni D, Lonardi F, Fusetti S, Nocini PF, Albanese M. Transnasal endoscopic and intra-oral combined approach for odontogenic cysts. Acta Otorhinolaryngol Ital. 2018;38(5):439-444. doi: 10.14639/0392-100X-1915.

14. Parvini P, Obreja K, Begic A, Schwarz F, Becker J, Sader R, Salti L. Decision-making in closure of oroantral communication and fistula. Int J Implant Dent. 2019;5(1):13. doi: 10.1186/s40729-019-0165-7.

15. Emes Y, Aga U, Cesur A, Soluk-Tekkesin M, Aybar B, Alatli C. Primary Closure of Oroantral Communication Using Pedicled Buccal Fat Pad Following Maxillary Cyst Enucleation. J Craniofac Surg. 2018;29(2):e131-e133. doi: 10.1097/ SCS.0000000000004213. 